\title{
Patient-centered care or osteopathic manipulative treatment as mediators of clinical outcomes in patients with chronic low back pain
}

https://doi.org/10.1515/jom-2021-0113

Received April 10, 2021; accepted June 16, 2021;

published online August 3, 2021

\begin{abstract}
Context: Patient-centered care is often considered a characteristic of osteopathic medicine, in addition to the use of osteopathic manipulative treatment (OMT) in such musculoskeletal conditions as low back pain.

Objectives: This study aimed to determine if patientcentered care or OMT are mediators of the clinical outcomes of osteopathic medicine in patients with chronic low back pain.
\end{abstract}

Methods: A comparative effectiveness study was conducted within the Pain Registry for Epidemiological, Clinical, and Interventional Studies and Innovation (PRECISION Pain Research Registry). Eligible patients met the diagnostic criteria recommended by the National Institutes of Health Task Force on Research Standards for Chronic Low Back Pain and completed four consecutive quarterly encounters between April 2016 and November 2020. The Consultation and Relational Empathy instrument for patient-centered care was used at the baseline encounter and OMT use was measured at the final encounter. The clinical outcome measures included low back pain intensity on a numerical rating scale (NRS) from 0 to 10, back-related functioning on the Roland-Morris Disability Questionnaire (RMDQ), and pain impact on the National Institutes of Health Minimum Dataset for Chronic Low Back Pain (NIH-MDS). A parallel multiple mediator model was used to compute the direct and indirect effects of osteopathic medicine in achieving each of the three clinical outcomes.

\footnotetext{
*Corresponding author: John C. Licciardone, DO, MS, MBA, Regents Professor, Osteopathic Research Center, Department of Family Medicine, University of North Texas Health Science Center, 3500 Camp Bowie Boulevard, Fort Worth, TX 76107, USA,

E-mail: john.licciardone@unthsc.edu

Subhash Aryal, PhD, School of Nursing, University of Pennsylvania, Philadelphia, PA, USA
}

Results: The 404 study patients had a mean age of 52.2 years (standard deviation, 13.1 years) and $288(71.3 \%)$ were female. The $88(21.8 \%)$ patients treated by osteopathic physicians reported more favorable scores for patient-centered care (mean, 41.3; 95\% CI 39.0-43.5) than patients treated by allopathic physicians (mean, 38.0; 95\% CI 36.8-39.3) ( $\mathrm{p}=0.02)$. Fifty-six (63.6\%) patients treated by osteopathic physicians used OMT. The age- and sex-adjusted outcomes for patients of osteopathic vs. allopathic physicians across all four encounters were: mean, 5.4; 95\% CI 5.0-5.7 vs. mean, 5.9; 95\% CI 5.7-6.1 on the NRS for pain intensity ( $\mathrm{p}=0.01$ ); mean, $11.3 ; 95 \%$ CI $10.1-12.6$ vs. mean, 14.0 ; $95 \%$ CI $13.3-14.7$ on the RMDQ for back-related disability ( $\mathrm{p}<0.001$ ); and mean, $26.8 ; 95 \%$ CI $24.9-28.7$ vs. mean, 30.1 ; $95 \%$ CI $29.1-31.1$ on the NIH-MDS for pain impact ( $\mathrm{p}=0.002)$. Patient-centered care did not mediate any outcome of osteopathic medicine, whereas OMT mediated better outcomes in low back pain intensity.

Conclusions: This appears to be the first study to simultaneously address both patient-centered care and OMT as potential mediators of the effect of osteopathic medicine in treating chronic pain. Patient-centered care did not mediate the effects of osteopathic medicine and OMT only mediated outcomes relating to low back pain intensity. More research is needed to identify other aspects of osteopathic medicine that mediate its beneficial effects in patients with chronic low back pain.

Keywords: back-related disability; chronic low back pain; mediation analysis; osteopathic manipulative treatment; osteopathic medicine; pain impact; pain intensity; pain research registry; patient-centered care.

A joint statement from the American Medical Association in conjunction with the American Osteopathic Association recently called for an effort to combat the mischaracterization of osteopathic physicians by media, celebrities, and companies [1]. It emphasized that osteopathic medicine is a distinctive branch of medical practice in the United States that involves a whole-person approach to care, including 
the use of osteopathic manipulative treatment (OMT) for such musculoskeletal conditions as low back pain. Similar claims about the distinctiveness of osteopathic medicine, which also focused on the dual tenets of a holistic or patientcentered approach to care and OMT, have been widely espoused for over two decades [2]. Data from the National Ambulatory Medical Care Survey have shown that low back pain historically has been a leading reason for patients to visit osteopathic physicians [3], and continues to be so today in primary care [4]. Based largely on systematic reviews and meta-analyses [5, 6], the only clinical practice guidelines issued by the American Osteopathic Association recommend that OMT be used when somatic dysfunction is the cause of or a contributing factor in low back pain $[7,8]$. The latter is defined as impaired or altered function of related components of the body framework system, including skeletal, arthrodial, and myofascial structures, and their related vascular, lymphatic, and neural elements [9]. Thus, low back pain represents an ideal condition to test the hypothesized distinctive aspects of osteopathic medicine. In particular, chronic low back pain enables a long-term assessment of the patient-centeredness of the ongoing physician-patient relationship as well as OMT. The purpose of this study was to use longitudinal data from a pain research registry to measure the clinical outcomes of patients with chronic low back pain and to determine if the effects of osteopathic medicine are mediated by either patient-centered care or OMT.

\section{Methods}

\section{Pain Registry for Epidemiological, Clinical, and Interventional Studies and Innovation}

Patients in this study were selected from the Pain Registry for Epidemiological, Clinical, and Interventional Studies and Innovation (PRECISION Pain Research Registry). The registry was established at the University of North Texas Health Science Center in 2016 to collect longitudinal data at quarterly encounters relating to chronic low back pain treatments and clinical outcomes [10]. Patients who reported being pregnant or institutionalized were not eligible to enroll in the registry. The registry transitioned to a digital research platform in 2020, which extended its reach to the 48 contiguous United States and District of Columbia using social media. The registry's platform has facilitated ongoing collection of clinical research data and the maintenance of standard operating procedures for clinical research during the COVID-19 pandemic [11], including the conduct of a randomized registry trial [12]. The registry was approved by the North Texas Regional Institutional Review Board (protocol 2015-169) and all patients provided written informed consent prior to enrollment.

\section{Eligibility criteria}

Patients were eligible for the study if they were 21-79 years of age at registry enrollment, had sufficient English-language proficiency to complete case report forms independently or with staff assistance, had a physician who regularly treated their low back pain, and had chronic low back pain according to diagnostic criteria established by the National Institutes of Health Task Force on Research Standards for Chronic Low Back Pain [13]. The latter require that patients report low back pain during at least the past 3-6 months, with a frequency of at least one-half of the days during the past 6 months. All eligible patients must have completed four consecutive quarterly encounters between April 2016 and November 2020, thereby representing 12 months of observation. Patients who reported physician crossover (i.e., being treated by an osteopathic physician at the first quarterly encounter and an allopathic physician at the final quarterly encounter, or vice-versa) were excluded from the study.

\section{Measurement of patient-centered care}

Patient-centered care was measured at the first quarterly encounter with the Consultation and Relational Empathy (CARE) instrument, which is comprised of 10 items relating to physician-patient interactions during medical care visits $[14,15]$. The CARE instrument has been validated as a measure of physician empathy-based consultation during the process of delivering primary care. In particular, one item specifically queries whether the physician is interested in the patient as "a whole person," while another item addresses physician "care and compassion." Total scores on the CARE instrument range from 10 to 50 , with higher scores reflective of a more patient-centered approach that is conducive to providing high-quality medical care for patients with chronic problems. Scores on individual items of the CARE instrument were multiplied by a factor of 10 to place them on the same scale as the total score for comparative purposes.

\section{Measurement of osteopathic manipulative treatment}

The use of commonly recommended treatments for chronic low back pain $[16,17]$ was measured at each quarterly encounter, including use of both non-pharmacological treatments (exercise therapy, yoga, massage therapy, spinal manipulation, acupuncture, and cognitive behavioral therapy) and pharmacological treatments such as nonsteroidal anti-inflammatory drugs (NSAIDs) and opioids. The use of OMT was determined with a trichotomous variable derived from the two items involving use of spinal manipulation and the type of physician provider. Patients who reported never using spinal manipulation at the final quarterly encounter were considered to have never used OMT (OMT $=0$ ), regardless of their physician type. Patients who reported using spinal manipulation were considered to have possibly used OMT (OMT=1) if they were treated by an allopathic physician. For example, it is possible that such patients may have received OMT from an osteopathic manipulative medicine specialist. The latter generally restrict their practices to providing OMT as a complement to primary care provided by other physicians. However, other health care professionals, such as chiropractors, may also provide such complementary spinal 
Table 1: Patient sociodemographic and clinical characteristics at the first quarterly encounter by physician type $(n=404){ }^{a}$

\begin{tabular}{|c|c|c|c|c|c|}
\hline \multirow[b]{3}{*}{ Characteristic } & \multirow{2}{*}{\multicolumn{2}{|c|}{$\frac{\text { Osteopathic physician }}{n=88}$}} & \multirow{2}{*}{\multicolumn{2}{|c|}{$\frac{\text { Allopathic physician }}{n=316}$}} & \multirow[b]{3}{*}{ p-Value } \\
\hline & & & & & \\
\hline & No. & $\%$ & No. & $\%$ & \\
\hline Age, years (mean, SD) & 53.2 & 14.3 & 52.0 & 12.7 & 0.45 \\
\hline Female sex & 66 & 75.0 & 222 & 70.3 & 0.38 \\
\hline \multicolumn{5}{|l|}{ Race } & 0.06 \\
\hline White & 73 & 83.0 & 215 & 68.0 & \\
\hline Black & 13 & 14.8 & 89 & 28.2 & \\
\hline Asian & 2 & 2.3 & 5 & 1.6 & \\
\hline American Indian/Alaska Native & 0 & 0.0 & 6 & 1.9 & \\
\hline Native Hawaiian/Pacific Islander & 0 & 0.0 & 1 & 0.3 & \\
\hline Hispanic ethnicity & 10 & 11.4 & 37 & 11.7 & 0.93 \\
\hline \multicolumn{5}{|l|}{ Educational level } & 0.051 \\
\hline Less than college degree & 44 & 50.0 & 201 & 63.6 & \\
\hline Bachelor's or Master's degree & 42 & 47.7 & 106 & 33.5 & \\
\hline Professional or doctoral degree & 2 & 2.3 & 9 & 2.8 & \\
\hline Ever lost work for 1 or more months due to low back pain & 25 & 28.4 & 127 & 40.2 & 0.04 \\
\hline \multicolumn{6}{|l|}{ benefits because of inability to work due to low back pain } \\
\hline Ever involved in a legal claim related to low back pain & 10 & 11.4 & 32 & 10.1 & 0.74 \\
\hline \multicolumn{5}{|l|}{ Cigarette smoking status } & 0.01 \\
\hline Never smoked & 53 & 60.2 & 158 & 50.0 & \\
\hline Former smoker & 31 & 35.2 & 106 & 33.5 & \\
\hline Current smoker & 4 & 4.5 & 52 & 16.5 & \\
\hline Body mass index, $\mathrm{kg} / \mathrm{m}^{2}$ (mean, SD) & 31.8 & 7.7 & 33.0 & 8.4 & 0.25 \\
\hline \multicolumn{6}{|l|}{ Medical conditions ever diagnosed } \\
\hline Herniated disc & 26 & 29.5 & 121 & 38.3 & 0.13 \\
\hline Sciatica & 32 & 36.4 & 123 & 38.9 & 0.66 \\
\hline Osteoarthritis & 24 & 27.3 & 110 & 34.8 & 0.18 \\
\hline Osteoporosis & 11 & 12.5 & 32 & 10.1 & 0.52 \\
\hline Hypertension & 32 & 36.4 & 132 & 41.8 & 0.36 \\
\hline Heart disease & 13 & 14.8 & 23 & 7.3 & 0.03 \\
\hline Diabetes mellitus & 16 & 18.2 & 60 & 19.0 & 0.86 \\
\hline Asthma & 22 & 25.0 & 87 & 27.5 & 0.64 \\
\hline Depression & 38 & 43.2 & 157 & 49.7 & 0.28 \\
\hline \multicolumn{5}{|l|}{ History of low back surgery } & 0.49 \\
\hline Yes, one surgery & 8 & 9.1 & 24 & 7.6 & \\
\hline Yes, more than one surgery & 3 & 3.4 & 21 & 6.6 & \\
\hline No & 77 & 87.5 & 271 & 85.8 & \\
\hline \multicolumn{6}{|l|}{ Ever used non-pharmacological treatments for low back pain } \\
\hline Exercise therapy & 44 & 50.0 & 194 & 61.4 & 0.055 \\
\hline Yoga & 25 & 28.4 & 79 & 25.0 & 0.52 \\
\hline Massage therapy & 45 & 51.1 & 150 & 47.5 & 0.54 \\
\hline Spinal manipulation & 58 & 65.9 & 117 & 37.0 & $<0.001$ \\
\hline Acupuncture & 19 & 21.6 & 45 & 14.2 & 0.09 \\
\hline Cognitive behavioral therapy & 6 & 6.8 & 30 & 9.5 & 0.44 \\
\hline \multicolumn{6}{|l|}{ Currently use pharmacological treatments for low back pain } \\
\hline Nonsteroidal anti-inflammatory drugs & 55 & 62.5 & 214 & 67.7 & 0.36 \\
\hline Opioids & 25 & 28.4 & 120 & 38.0 & 0.10 \\
\hline
\end{tabular}

${ }^{\mathrm{a}}$ Table entries for characteristics are No. (\%), unless otherwise specified.

\section{Clinical outcome measures}

manipulation. Patients who reported using spinal manipulation were considered to have used OMT (OMT=2) if they were treated by an osteopathic physician.
The clinical outcomes (low back pain intensity, back-related functioning, and pain impact) were measured at each quarterly encounter. 


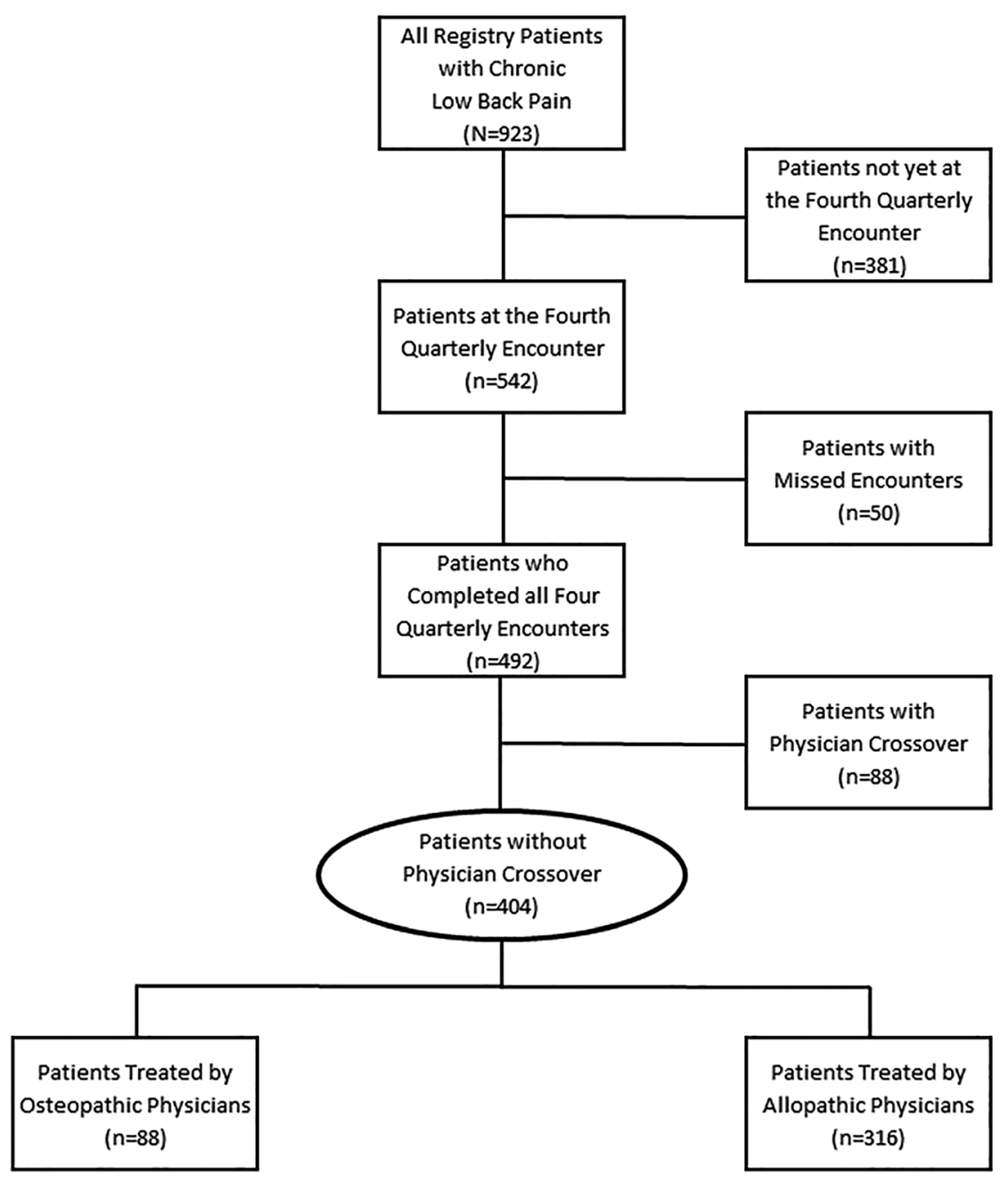

Figure 1: Flow diagram for selection of study patients.
Low back pain intensity was measured with an 11-point numerical rating scale (NRS) ranging from 0 ("no pain") to 10 ("worst pain") for the average pain level over the past 7 days. Back-related functioning was measured with the Roland-Morris Disability Questionnaire (RMDQ), which assessed back-related disability on the encounter date [18]. Scores on the RMDQ range from 0 to 24, with higher scores indicating greater levels of disability. Pain impact was measured using nine items from the National Institutes of Health Minimum Dataset for Chronic Low Back Pain (NIH-MDS), including scales for physical function and pain interference with activities [13]. The latter scales were derived from the Patient-Reported Outcomes Measurement Information System for health-related quality of life [19]. Higher scores represent worse outcomes on the pain impact measure.

\section{Statistical analysis}

Patient characteristics and clinical measures were summarized using the no. (\%) for dichotomous or categorical variables and the mean (standard deviation [SD]) for continuous variables. Comparisons of these measures according to physician type were performed with contingency table methods and the Student's $t$-test, respectively. Repeated measures analysis of variance was used to compare each of the clinical outcomes over the four quarterly encounters by physician type, with patient age and sex entered as covariates to control for potential confounding. Mediation analyses were performed to determine if patient-centered care or OMT mediated the effects of osteopathic medicine in achieving any of the clinical outcomes. These analyses were performed with the PROCESS program [20], a regression-based approach that enables computation of the mean effects of osteopathic medicine and 95\% CIs. Physician type was entered as the independent variable and the grand mean of the four measures at each quarterly encounter was entered as the dependent variable in each of the respective analyses for the three clinical outcomes. A parallel multiple mediator model was used to compute the direct and indirect (i.e., mediated) effects of osteopathic medicine with 50,000 resamples used to compute percentile bootstrap 95\% CIs for patient-centered care and OMT, while controlling for patient age and sex. All database management and other statistical analyses were performed with the IBM SPSS Statistics software (Version 25). Hypotheses were assessed at the 0.05 level of statistical significance using 2-sided tests.

\section{Results}

A total of 404 patients were included in the study, with 88 (21.8\%) treated by osteopathic physicians and 316 (78.2\%) 


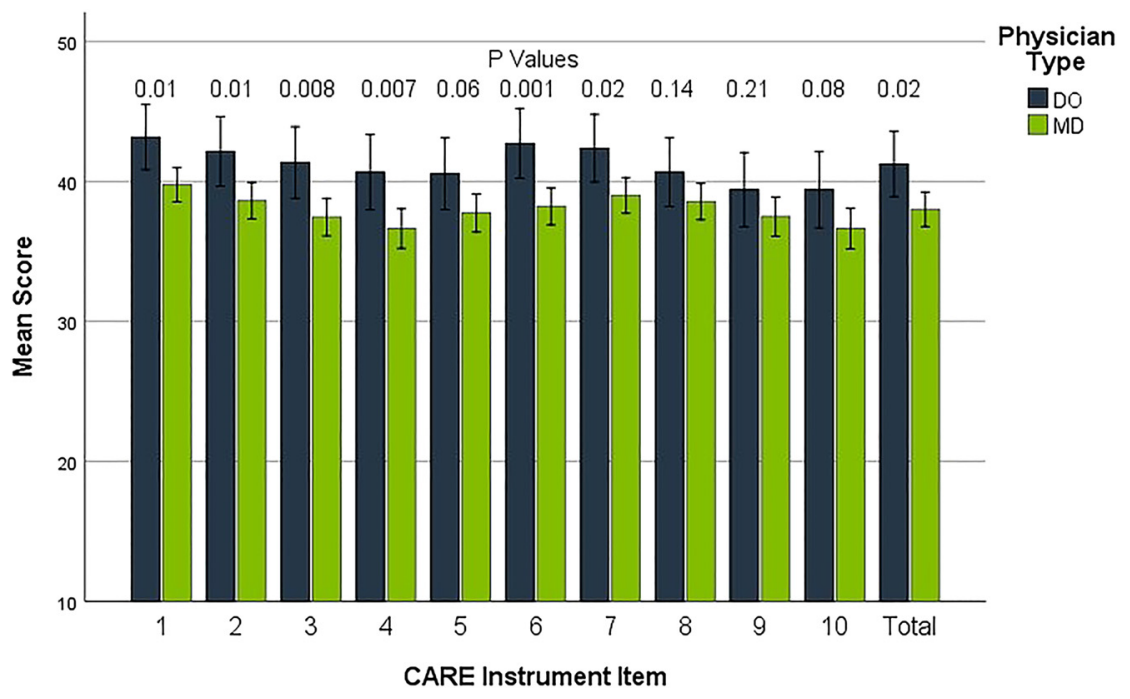

Figure 2: Measures on the Consultation and Relational Empathy (CARE) instrument according to physician type. Error bars represent $95 \%$ confidence intervals. $p$-values were computed using the Student's $t$-test based on 88 patients treated by osteopathic physicians (DOs) and 316 patients treated by allopathic physicians (MDs). The CARE instrument items measure the following patient perceptions of their physician: 1. Making you feel at ease; 2 . Letting you tell your "story"; 3 . Really listening; 4. Being interested in you as a whole person; 5 . Fully understanding your concerns; 6 . Showing care and compassion; 7. Being positive; 8. Explaining things clearly; 9. Helping you to take control; 10. Making a plan of action with you. Scores range from 10 to 50, with higher scores representing a more patient-centered approach.

treated by allopathic physicians (Figure 1). Overall, the mean age of patients was 52.2 years (SD, 13.1 years) and 288 $(71.3 \%)$ were female (Table 1$)$. Patients treated by either type of physician were generally comparable. However, patients treated by osteopathic physicians were less likely to report ever having a work loss of 1 or more months due to low back pain or to be current cigarette smokers, but they were more likely to report a history of heart disease and ever having used spinal manipulation. There were no differences according to physician type in ever having used exercise therapy, yoga, massage therapy, acupuncture, or cognitive behavioral therapy, or in currently using NSAIDs or opioids for low back pain. The outcome measures over all four quarterly encounters were: mean, 5.8; 95\% CI 5.6-6.0 on the NRS for low back pain intensity; mean, 13.4; 95\% CI 12.8-14.0 on the RMDQ for back-related functioning; and mean, 29.4; 95\% CI 28.5-30.3 on the NIH-MDS for pain impact.

Patients treated by osteopathic physicians reported higher total scores on the CARE instrument (mean, 41.3; 95\% CI 39.0-43.5) than patients treated by allopathic physicians (mean, 38.0; 95\% CI 36.8-39.3) ( $\mathrm{p}=0.02$ ) (Figure 2). Osteopathic physicians received higher scores than allopathic physicians on six of the 10 CARE instrument items, including those that measured care and compassion (mean, 42.7; 95\% CI 40.4-45.1 vs. mean, 38.2; 95\% CI 36.9-39.6; $\mathrm{p}=0.001$ ) and being interested in the patient as a whole person (mean, 40.7; 95\% CI 38.1-43.2 vs. mean, 36.6; 95\% CI
35.2-38.1; $\mathrm{p}=0.007)$. Overall, 211 (52.2\%) patients had never used OMT. A total of $56(63.6 \%)$ patients treated by osteopathic physicians reported having used OMT for low back pain at the final quarterly encounter, compared with 137 (43.4\%) patients treated by allopathic physicians who may possibly have used OMT.

Patients treated by osteopathic physicians reported lower scores for low back pain intensity, back-related disability, and pain impact than patients treated by allopathic physicians over the four quarterly encounters (Figure 3). The corresponding age- and sex-adjusted main effects outcomes for patients of osteopathic vs. allopathic physicians during this period of observation were: mean, 5.4; 95\% CI 5.0-5.7 vs. mean, 5.9; 95\% CI 5.7-6.1 ( $\mathrm{p}=0.01)$ on the NRS for low back pain intensity; mean, 11.3; 95\% CI $10.1-12.6$ vs. mean, 14.0 ; $95 \%$ CI $13.3-14.7$ ( $\mathrm{p}<0.001$ ) on the RMDQ for back-related disability; and mean, 26.8; 95\% CI 24.9-28.7 vs. mean, 30.1; 95\% CI 29.1-31.1 ( $\mathrm{p}=0.002)$ on the NIH-MDS for pain impact. There was also a significant physician type $\times$ time interaction for low back pain intensity, wherein pain decreased over time in patients treated by osteopathic physicians but remained stable in patients treated by allopathic physicians $(\mathrm{p}=0.03)$.

Patient-centered care did not mediate the effects of osteopathic medicine in achieving any of the clinical outcomes. The use of OMT was a significant mediator of the effect of osteopathic medicine on low back pain intensity ( $\beta$-coefficient, $-0.27 ; 95 \%$ CI -0.52 to -0.04 
A

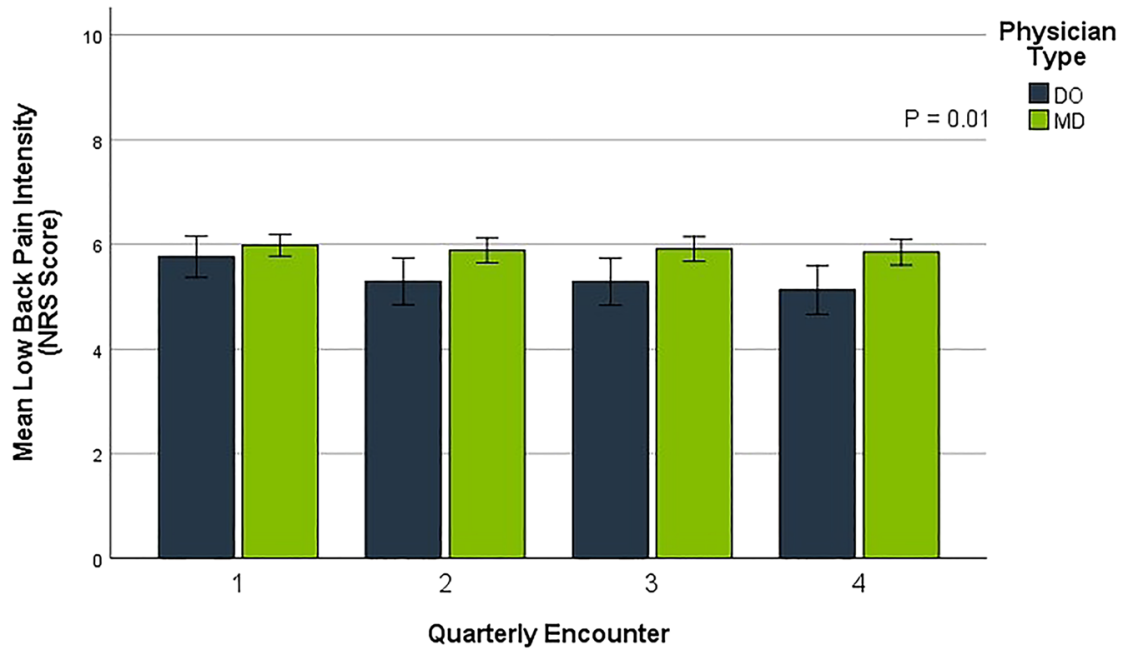

B

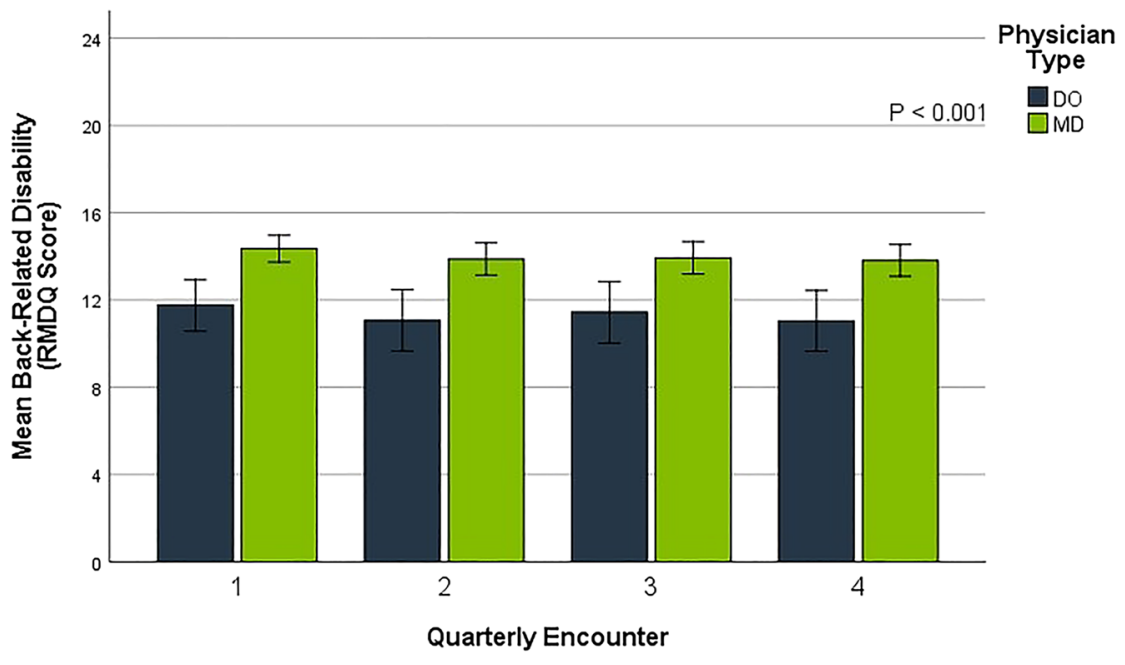

C

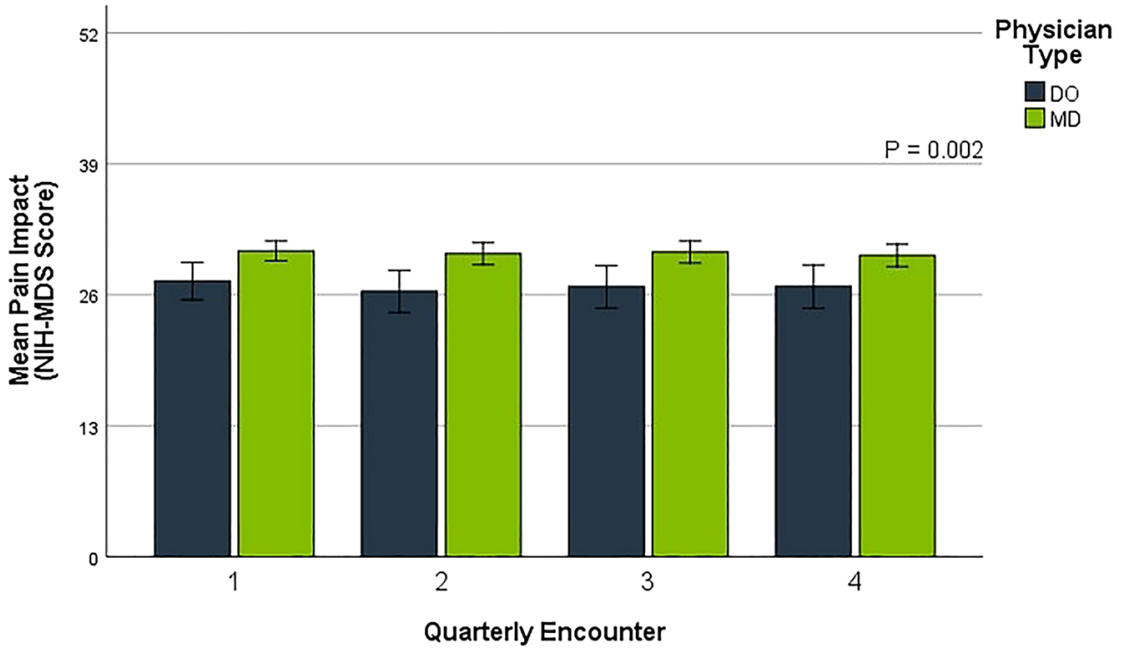

Figure 3: Clinical outcomes over time according to physician type. Outcomes include (A) low back pain intensity, (B) back-related disability, and (C) pain impact. Error bars represent $95 \%$ confidence intervals. $p$-values were computed using repeated measures analysis of variance based on 88 patients treated by osteopathic physicians (DOs) and 316 patients treated by allopathic physicians (MDs). NRS, numerical rating scale; RMDQ, Roland-Morris Disability Questionnaire; NIH-MDS, National Institutes of Health Minimum Dataset for Chronic Low Back Pain. Higher scores represent worse outcomes on each measure. A significant physician type $\times$ time interaction was observed for low back pain intensity $(p=0.03)$. 
Table 2: Regression coefficient estimates for the direct and indirect effects of osteopathic medicine on clinical outcomes ( $n=404){ }^{a}$

\begin{tabular}{|c|c|c|c|}
\hline \multirow{3}{*}{ Effect of osteopathic medicine } & \multicolumn{3}{|c|}{ Clinical outcome } \\
\hline & \multicolumn{3}{|c|}{ Low back pain intensity } \\
\hline & $\boldsymbol{\beta}$ & $95 \% \mathrm{Cl}$ & p-Value \\
\hline Total effect of osteopathic medicine & -0.54 & -0.97 to -0.11 & 0.01 \\
\hline Total direct effect of osteopathic medicine & -0.24 & -0.73 to 0.25 & 0.33 \\
\hline Total indirect effect of osteopathic medicine & -0.30 & -0.56 to -0.06 & 0.02 \\
\hline Patient-centered care & -0.03 & -0.09 to 0.02 & 0.34 \\
\hline \multirow[t]{3}{*}{ Osteopathic manipulative treatment } & -0.27 & -0.52 to -0.04 & 0.03 \\
\hline & \multicolumn{3}{|c|}{ Back-related disability } \\
\hline & $\beta$ & $95 \% \mathrm{Cl}$ & p-Value \\
\hline Total effect of osteopathic medicine & -2.66 & -4.08 to -1.25 & $<0.001$ \\
\hline Total direct effect of osteopathic medicine & -2.32 & -3.95 to -0.69 & 0.006 \\
\hline Total indirect effect of osteopathic medicine & -0.35 & -1.15 to 0.49 & 0.40 \\
\hline Patient-centered care & -0.16 & -0.38 to 0.02 & 0.14 \\
\hline \multirow[t]{3}{*}{ Osteopathic manipulative treatment } & -0.19 & -0.97 to 0.61 & 0.63 \\
\hline & \multicolumn{3}{|c|}{ Pain impact } \\
\hline & $\beta$ & $95 \% \mathrm{Cl}$ & p-Value \\
\hline Total effect of osteopathic medicine & -3.32 & -5.45 to -1.18 & 0.002 \\
\hline Total direct effect of osteopathic medicine & -2.71 & -5.17 to -0.24 & 0.03 \\
\hline Total indirect effect of osteopathic medicine & -0.61 & -1.80 to 0.64 & 0.33 \\
\hline Patient-centered care & -0.26 & -0.64 to 0.02 & 0.12 \\
\hline Osteopathic manipulative treatment & -0.35 & -1.49 to 0.84 & 0.56 \\
\hline
\end{tabular}

${ }^{*} p$-values were computed using mediation analysis based on 88 patients treated by osteopathic physicians and 316 patients treated by allopathic physicians. Clinical outcomes were measured over four quarterly encounters using a numerical rating scale for low back pain intensity, the Roland-Morris Disability Questionnaire for back-related disability, and the National Institutes of Health Minimum Dataset items for pain impact. Negative $\beta$-coefficients represent more favorable outcomes with osteopathic medicine (i.e., lower scores for low back pain intensity, back-related disability, and pain impact). The total indirect effect of osteopathic medicine is that which is attributable to the two potential mediators in the regression models.

( $\mathrm{p}=0.03)$ (Table 2). Osteopathic manipulative treatment mediated about one-half of the total effect of osteopathic medicine relating to low back pain intensity based on the regression model coefficients. However, OMT did not mediate clinical outcomes relating to back-related functioning or pain impact.

\section{Discussion}

There are over 150,000 osteopathic physicians and medical students in the United States [21], including almost 22,000 osteopathic resident physicians in programs accredited by the Accreditation Council for Graduate Medical Education and combined specialty programs [22]. Yet, fundamental misrepresentations of osteopathic physicians often occur, such as those involving televised mass media reports that questioned why an "osteopath" led the multidisciplinary team that treated President Trump for COVID-19 and implied that he was not an "actual doctor." [23] This prompted the American Medical Association and American Osteopathic Association to combat such misrepresentations in their joint statement by focusing on the distinct and equivalent training of osteopathic physicians [1]. However, relatively little research has addressed whether such distinctiveness remains after osteopathic physicians complete their training and enter the workforce. This study begins to provide evidence in response to this question by examining the osteopathic approach to chronic low back pain, which may be considered a "best-case scenario" for demonstrating its distinctiveness and corresponding effects on clinical outcomes. Indeed, patients rated osteopathic physicians more highly than allopathic physicians for overall patient-centered care and in such aspects as "care and compassion" and treating the "whole person." Also, not surprisingly, a majority of patients treated by osteopathic physicians had received OMT for low back pain.

Virtually all previous research on osteopathic medicine for chronic low back pain has focused on the efficacy of OMT. The two largest trials, which both followed patients for only 12 weeks, found conflicting results relating to low back pain intensity. One trial involving 155 patients failed to observe significant improvements in low back pain intensity or back-related functioning with OMT [24]. A later trial 
involving 455 patients found significant improvement in low back pain intensity with OMT, but not in back-related functioning or general health [25]. The most recent systematic review and meta-analysis that included these and other trials found that OMT significantly improved low back pain intensity and functional status in patients with chronic low back pain [6]. In our study, patients treated by osteopathic physicians reported significantly lower scores for low back pain intensity, back-related disability, and pain impact than patients treated by allopathic physicians. All three of these physician group differences in outcomes are clinically important based on effect size criteria [26]. Such longitudinal outcomes in a pragmatic study within a real-world setting demonstrate effects of osteopathic medicine that have not always been evident during short-term efficacy trials of OMT.

Although osteopathic physicians were reported to have a more patient-centered approach to medical care than allopathic physicians, it was not a mediator of the effect of osteopathic medicine in achieving any of the three clinical outcomes in our study. This is somewhat surprising given that the osteopathic patient-centered approach is closely aligned with the biopsychosocial model of pain [27, 28]. Perhaps more fittingly, osteopathic principles [29], and the American Osteopathic Association clinical practice guidelines in particular $[7,8]$, stress the importance of using OMT to treat somatic dysfunction when it is the cause of or a contributing factor in low back pain. Somatic dysfunction is characterized by positional asymmetry, restricted range of motion, tissue texture abnormalities, or tenderness, which may include pain elicited through palpation [9]. Osteopathic manipulative treatment is used to alleviate somatic dysfunction by applying manually guided forces to improve physiologic function and support homeostasis. It is unclear why OMT mediated better outcomes in low back pain intensity, but not in back-related functioning or pain impact, because OMT is more often used to treat restricted range of motion than tenderness or pain [30].

There were both advantages and limitations of this study. To our knowledge, this is the first study to simultaneously address both patient-centered care and OMT as potential mediators of the effect of osteopathic medicine in treating chronic pain. It was conducted within a pain research registry, thereby providing a well-defined population for longitudinal observation using the case definition and clinical outcome measures recommended by the National Institutes of Health Task Force on Research Standards for Chronic Low Back Pain [13]. Although formal mediation analyses were performed [20], there were limitations of this approach. First, OMT was only assessed with a categorical measure using the available registry data rather than with a more robust continuous measure. This categorical measure was sufficient to identify OMT as a mediator of the effect of osteopathic medicine on low back pain intensity; however, it may have limited our ability to detect mediation of the other clinical outcomes. Moreover, this measure based on use of spinal manipulation and physician type may not have been entirely specific for OMT (i.e., for OMT provided by osteopathic physicians vs. spinal manipulation provided by other health care professionals). Second, although our sample of 404 patients was sufficiently large to detect the significant effects of osteopathic medicine on each of the three clinical outcomes, an a-priori sample size was not computed for the mediation analyses because of the scarcity of relevant data on the magnitude of potential effects of the hypothesized mediators [20]. Nevertheless, based on empirical sample size estimates involving percentile bootstrap tests such as ours, the sample size was sufficiently large to achieve or surpass approximately $80 \%$ statistical power under most likely scenarios [31].

\section{Conclusions}

Patients with chronic low back pain reported that osteopathic physicians provided a more patient-centered approach to medical care than allopathic physicians and often used OMT. These patients also reported better long-term outcomes relating to low back pain intensity, back-related functioning, and pain impact. However, patient-centered care did not mediate any of the effects of osteopathic medicine. The use of OMT mediated the effect of osteopathic medicine in achieving better outcomes in low back pain intensity, but not in the other two domains. More research is needed to identify other aspects of osteopathic medicine that mediate its beneficial effects in patients with chronic low back pain.

Research funding: This study was supported in part by grants from the Osteopathic Heritage Foundation and the American Osteopathic Association. The funding organizations had no part in the design and conduct of the study; collection, management, analysis, and interpretation of the data; preparation, review, or approval of the manuscript; and decision to submit the manuscript for publication.

Author contributions: Both authors provided substantial contributions to conception and design, acquisition of 
data, or analysis and interpretation of data; both authors drafted the article or revised it critically for important intellectual content; both authors gave final approval of the version of the article to be published; and both authors agree to be accountable for all aspects of the work in ensuring that questions related to the accuracy or integrity of any part of the work are appropriately investigated and resolved.

Competing interests: None reported.

Informed consent: All patients provided written informed consent prior to enrollment.

Ethical approval: The registry was approved by the North Texas Regional Institutional Review Board (protocol 2015-169).

\section{References}

1. American Medical Association. AOA and AMA stand against misrepresentation of osteopathic physicians. Available from: https://www.ama-assn.org/press-center/ama-statements/aoaand-ama-stand-against-misrepresentation-osteopathicphysicians [Accessed 23 Nov 2020].

2. Howell JD. The paradox of osteopathy. N Engl J Med 1999;341: 1465-8.

3. Cypress BK. Characteristics of physician visits for back symptoms: a national perspective. Am J Public Health 1983;73:389-95.

4. Licciardone JC. A national study of primary care provided by osteopathic physicians. J Am Osteopath Assoc 2015;115:704-13.

5. Licciardone JC, Brimhall AK, King LN. Osteopathic manipulative treatment for low back pain: a systematic review and metaanalysis of randomized controlled trials. BMC Musculoskelet Disord 2005;6:43.

6. Franke H, Franke JD, Fryer G. Osteopathic manipulative treatment for nonspecific low back pain: a systematic review and metaanalysis. BMC Musculoskelet Disord 2014;15:286.

7. Clinical Guideline Subcommittee on Low Back Pain. American Osteopathic Association guidelines for osteopathic manipulative treatment (OMT) for patients with low back pain. J Am Osteopath Assoc 2010;110:653-66.

8. Task Force on the Low Back Pain Clinical Practice Guidelines. American Osteopathic Association Guidelines for Osteopathic Manipulative Treatment (OMT) for Patients with Low Back Pain. J Am Osteopath Assoc. 2016;116:536-49.

9. Glossary of osteopathic terminology. In: Seffinger MA, Hruby R, Willard FH, Licciardone J, editors Foundations of osteopathic medicine: philosophy, science, clinical applications, and research, 4th ed. Philadelphia, PA: Wolters Kluwer; 2018:1563-94 pp.

10. Licciardone JC, Gatchel RJ, Phillips N, Aryal S. The Pain Registry for Epidemiological, Clinical, and Interventional Studies and Innovation (PRECISION): registry overview and protocol for a propensity score-matched study of opioid prescribing in patients with low back pain. J Pain Res 2018;11:1751-60.

11. Licciardone JC. Demographic characteristics associated with utilization of noninvasive treatments for chronic low back pain and related clinical outcomes during the COVID-19 pandemic in the United States. J Am Board Fam Med 2021;34(Suppl):S77-84.
12. Licciardone JC, Pandya V. Feasibility trial of an eHealth intervention for health-related quality of life: implications for managing patients with chronic pain during the COVID-19 pandemic. Healthcare (Basel) 2020;8:381.

13. Deyo RA, Dworkin SF, Amtmann D, Andersson G, Borenstein D, Carragee E, et al. Report of the NIH Task Force on research standards for chronic low back pain. J Pain 2014;15:569-85.

14. Mercer SW, Maxwell M, Heaney D, Watt GC. The consultation and relational empathy (CARE) measure: development and preliminary validation and reliability of an empathy-based consultation process measure. Fam Pract 2004;21:699-705.

15. Mercer SW, McConnachie A, Maxwell M, Heaney D, Watt GC. Relevance and practical use of the Consultation and Relational Empathy (CARE) Measure in general practice. Fam Pract 2005;22: 328-34.

16. Dowell D, Haegerich TM, Chou R. CDC Guideline for Prescribing Opioids for Chronic Pain-United States, 2016. MMWR Recomm Rep 2016;65:1-49.

17. Qaseem A, Wilt TJ, McLean RM, Forciea MA. Clinical Guidelines Committee of the American College of Physicians. Noninvasive treatments for acute, subacute, and chronic low back pain: a clinical practice guideline from the American College of Physicians. Ann Intern Med 2017;166:514-30.

18. Roland M, Morris R. A study of the natural history of back pain. Part I: development of a reliable and sensitive measure of disability in low-back pain. Spine 1983;8:141-4.

19. PROMIS. Adult profile instruments. Evanston, IL: Northwestern University; 2015.

20. Hayes AF. Introduction to mediation, moderation, and conditional process analysis: a regression-based approach. New York: The Guilford Press; 2018.

21. American Osteopathic Association. Osteopathic Medical Profession Report 2019. Available from: https://osteopathic.org/ wp-content/uploads/OMP2019-Report_Web_FINAL.pdf [Accessed 5 Dec 2020].

22. Brotherton SE, Etzel SI. Graduate medical education, 2019-2020. J Am Med Assoc 2020;324:1230-50.

23. Raymond R. Osteopathic medical profession fights back after CNN, MSNBC mischaracterize DOs. Available from: https:// thedo.osteopathic.org/2020/10/osteopathic-medicalprofession-fights-back-after-major-news-outletsmischaracterize-dos/ [Accessed 10 Oct 2020].

24. Andersson GB, Lucente T, Davis AM, Kappler RE, Lipton JA, Leurgans S. A comparison of osteopathic spinal manipulation with standard care for patients with low back pain. N Engl J Med 1999;341:1426-31.

25. Licciardone JC, Minotti DE, Gatchel RJ, Kearns CM, Singh KP. Osteopathic manual treatment and ultrasound therapy for chronic low back pain: a randomized controlled trial. Ann Fam Med 2013;11:122-9.

26. Faraone SV. Interpreting estimates of treatment effects: implications for managed care. P T 2008;33:700-11.

27. Gatchel RJ. Comorbidity of chronic pain and mental health disorders: the biopsychosocial perspective. Am Psychol 2004;59: 795-805.

28. Minotti D, Licciardone JC, Kearns C, Gatchel RJ. Osteopathic medicine: approach to pain management. Pract Pain Manag 2010;10:28-38.

29. Seffinger MA, Hruby RJ, Rogers FJ, et al. Philosophy of osteopathic medicine. In: Seffinger MA, Hruby R, Willard FH, Licciardone J, 
editors. Foundations of osteopathic medicine: philosophy, science, clinical applications, and research, 4th ed. Philadelphia, PA: Wolters Kluwer; 2018:2-18 pp.

30. Licciardone JC, Kearns CM, King HH, Seffinger MA, Crow WT, Zajac $P$, et al. Somatic dysfunction and use of osteopathic manual treatment techniques during ambulatory medical care visits: a CONCORD-PBRN study. J Am Osteopath Assoc 2014;114: 344-54.

31. Fritz MS, Mackinnon DP. Required sample size to detect the mediated effect. Psychol Sci 2007;18:233-9. 